\title{
Multipactor discharge on metals and dielectrics: Historical review and recent theories*
}

\author{
R. A. Kishek ${ }^{\dagger, a)}$ \\ Institute for Plasma Research, ERB\#223, University of Maryland, College Park, Maryland 20742 \\ Y. Y. Lau, b) L. K. Ang, A. Valfells, and R. M. Gilgenbach \\ Department of Nuclear Engineering and Radiological Sciences, University of Michigan, Ann Arbor, \\ Michigan 48109-2104
}

(Received 18 November 1997; accepted 12 January 1998)

\begin{abstract}
This paper reviews the history of multipactor discharge theory, focusing on recent models of multipactor accessibility and saturation. Two cases are treated in detail: That of a first-order, two-surface multipactor, and that of a single-surface multipactor on a dielectric. In both cases, susceptibility curves are constructed to indicate the regions of external parameter space where multipactor is likely to occur, taking into account the dependence on surface materials, and the effects of space charge and cavity loading. In the case of a dielectric, multipactor is found to deliver about $1 \%$ of the rf power to the surface. The two cases are contrasted in light of experimental observations. (C) 1998 American Institute of Physics. [S1070-664X(98)92205-4]
\end{abstract}

\section{INTRODUCTION}

Multipactor discharge is a resonant vacuum discharge frequently observed in microwave systems such as radio frequency (rf) windows, ${ }^{1-6}$ accelerator structures,${ }^{7,8}$ microwave tubes and devices, ${ }^{6,9-12}$ and rf satellite payloads. ${ }^{13-15}$ The discharge can occur for a wide range of frequencies, from the $\mathrm{MHz}$ range to tens of $\mathrm{GHz}$, and in a wide array of geometries. The underlying mechanism behind the multipactor discharge is an avalanche caused by secondary electron emission. Primary electrons accelerated by rf fields can impact a surface and release a larger number of secondary electrons, which may in turn be accelerated by the rf fields and made to impact again, releasing even more electrons, and so on. The discharge can take place on a single surface or between two surfaces.

Multipactor is usually undesirable. It can dissipate substantial amounts of energy fed into microwave cavities, thus lengthening the conditioning process. It can detune a microwave signal (with detrimental consequences for space communications systems). The discharge also heats the surface, possibly increasing noise levels in crossed-field devices and perhaps causing damage. Through various mechanisms multipactor may result in window breakage, destroying the vacuum. In some circumstances (see Ref. 12), multipactor may even induce vacuum breakdown. It is worth noting that, despite all of the above, there is a growing number of applications for the discharge. For example, the inherent resonant bunching in multipactor has been explored for use in electron gun technology. ${ }^{16-18}$ The capability of multipactor to induce breakdown is under study for application in plasma display technology, while its capability to dissipate energy has been

*Paper kWeaI2-6 Bull. Am. Phys. Soc. 42, 1949 (1997).

'Invited speaker.

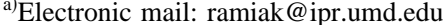

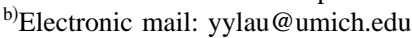

used for the protection of sensitive receivers.

In this paper, we review recent advances at the University of Michigan in the theoretical understanding of multipactor. The discharge is modeled from first principles, taking into account both space charge forces and interaction with the structure (loading and detuning). To our knowledge, this is the first theory that includes this interaction between the multipactor discharge and the surrounding rf structure. Our theory employs a realistic model of secondary electron emission for various surface materials. To put the recent theories in their proper context we start with a brief historical review of multipactor theory (Sec. II). Afterwards we describe the recent theories on accessibility and saturation of multipactor discharge. The presentation is divided into two sections: one dealing with two-surface multipactor (Sec. III), and another concentrating on the special, yet commonplace, case of single-surface multipactor on dielectrics (Sec. IV). The two cases are contrasted in the conclusion.

\section{HISTORICAL REVIEW ${ }^{\mathbf{1 9}}$}

Multipactor has been observed as early as 1924 by the Guttons (see Ref. 20), but was not identified until Farnsworth a decade later, who coined the name "multipactor" (from "AC Electron Multiplier") for an amplifier he invented based on the discharge (see Ref. 21). Observations of multipactor in gaseous breakdown experiments in 1930s and 1940s led to many early theories. Most notable are those of Daniellson, and of Henneburg et al., in 1936 (see Ref. 20). The latter derive the resonance condition on the transit time for electrons emitted with zero initial velocity, and also identify single particle phase focusing and stability of the discharge.

Perhaps the first systematic study of multipactor came from Gill and von Engel of Oxford University in the 1940s, ${ }^{22}$ in which they experimentally outlined part of the region susceptible to multipactor and, in conjunction, advanced a theory that recognized the sensitivity of the solution to a 
nonzero emission velocity of secondaries. In order to avoid the complications of a distribution of random emission velocities, Gill and von Engel introduced the ad hoc assumption that a parameter $k$, equal to the impact velocity of the primaries relative to the emission velocity of secondaries, is constant. There is no physical basis for this assumption. Furthermore, Gill and von Engel's attempt at using the theory to interpret their experimental results was flawed, and it was not until Hatch and Williams reformulated the theory in the 1950s to explain their own multipactor experiments ${ }^{20}$ that reasonable agreement was obtained. Since Hatch and Williams retained the constant " $k$ " assumption of Gill and von Engel, the modified theory became known as the "constant$k$ " theory and for decades remained the classic theory on the accessibility of multipactor, ${ }^{23,24}$ because of its utility in constructing susceptibility curves.

More recently, Vaughan ${ }^{21}$ has promoted an alternative to the constant $k$ theory, based rather on that by Henneburg et al., and derived from first principles. Vaughan's theory replaces the baseless assumption of constant $k$ with the more realistic assumption of a monoenergetic nonzero initial velocity. Other researchers ${ }^{19,25-30}$ have since then adopted Vaughan's theory. Riyopoulos et al. extended it to include a crossed-magnetic field. In Sec. III we use this theory in deriving the susceptibility curve, and extend it to include the effects of materials and of cavity loading.

In the decades following the rf breakdown experiments of Hatch and Williams, multipactor proliferated in all types of $\mathrm{rf}$ devices, and its suppression became a major concern. Many of the advances in understanding multipactor and the suppression techniques, however, remained proprietary to industries, and hence relatively little survives in the open literature. Currently, there are three general approaches for suppressing multipactor: conditioning of the components by the discharge, ${ }^{6}$ coatings and other "surface treatments,",6,8,11,31,32 and geometrical modifications. ${ }^{10,21,33-35}$ Conditioning is a lengthy process that depends on the discharge affecting the surface characteristics enough to quench itself. In general, surface treatments are aimed at reducing the effective secondary electron yield to inhibit the growth of multipactor, but unfortunately cannot be applied in all situations and are likely to degrade over time. ${ }^{21}$ The most promising method so far has been modifying the geometry (e.g., moving the location of a $\mathrm{rf}$ window ${ }^{21,35}$ ), with the goal of altering the trajectories of electrons so as to eliminate multipactor.

Despite the wealth of experimental data, the advances in multipactor theory moved at a much slower pace. The availability of more powerful computer platforms led to the emergence of many computer codes to model multipactor and aid in designing multipactor-free structures. ${ }^{8,33,34}$ Yet even today, the multipactor codes are limited to calculating singleelectron trajectories subject to a given rf field. Only recently are particle-in-cell (PIC) codes being applied to multipactor. ${ }^{18,26,27,36}$ The accessibility theories have also been extended to various types of geometries, such as single-surface ${ }^{1}$ or coaxial $^{37-39}$ geometries. Single-surface multipactor on dielectrics poses a special problem, ${ }^{1-5}$ and

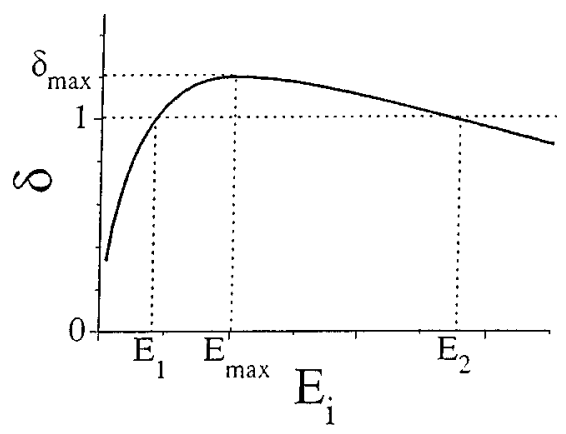

FIG. 1. Dependence of secondary electron yield on impact energy.

until recently, few satisfactory theories have been developed..$^{40,41}$

Neither the trajectory computations nor the accessibility theories say anything of the saturation level of the multipactor current or the time scale over which multipactor evolves. Many early researchers have suspected that debunching due to space charge forces can saturate the multipactor, ${ }^{1,16,17,22}$ but it was not until Vaughan in 1988 that anyone attempted to analytically estimate the level of saturation. ${ }^{21} \mathrm{Mako}^{18}$ and Riyopoulos $^{27}$ also assumed space charge debunching of the multipactor to be the cause of saturation, although each treated this effect somewhat differently. None of these saturation theories accounted in any way for the important processes of loading and detuning of rf structures by the multipactor. In Sec. III B we shall look at the first theory to explore this loading effect in resonant structures, while in Sec. IV we shall look at the first complete theory on accessibility and saturation of multipactor on dielectric windows.

\section{TWO-SURFACE MULTIPACTOR ON METALS}

In this section, we address the subject of a multipactor discharge between two metallic surfaces. For simplicity, we assume two parallel plane surfaces, separated by a gap of width $D$. A perpendicular of electric field, of the form $\left(V_{g 0} / D\right) \sin (2 \pi f t+\theta)$, where $f$ is the frequency of the $\mathrm{rf}$ and $V_{g 0}$ is the amplitude of the voltage across the gap, accelerates electrons inside the gap. Upon impact, secondaries are released with a monoenergetic nonzero initial velocity of a few eV (in Sec. IV we further include a random distribution of emission velocities). The secondary electron yield, $\delta$, is a function of impact energy and angle of the primary electron. ${ }^{42}$ In this paper, we adopt Vaughan's empirical formula for the yield. ${ }^{43}$ The dependence on impact energy is illustrated in Fig. 1 where the yield as a function of impact energy is determined by the two material-dependent parameters $E_{\max }$ and $\delta_{\max }$. These two parameters determine two energies for which the yield is unity, called the first and second cross-over points, $E_{1}$ and $E_{2}$, respectively (shown in the figure). Clearly, multipactor grows only for impact energies in between $E_{1}$ and $E_{2}$, where $\delta>1$.

\section{A. Susceptibility curves}

For the multipactor discharge to be sustained, electrons just released from one plate must be accelerated strongly enough to reach the other plate, and do so at a time when the 
field has reversed (or is close to doing so) in order that the secondary electrons released from the impact also be accelerated. This consideration leads to a condition on the transit time of the electrons, ${ }^{20,21,25}$ namely, that the transit time should be near an odd number, $N$, of $\frac{1}{2}$ rf cycles for multipactor to take place, where the number $N$ is called the order of the multipactor. This transit time condition (or synchrony condition) relates the rf voltage and frequency to the geometry, allowing us to map the regions of external parameter space in which multipactor is possible. Such maps are called susceptibility curves in the literature (e.g., Fig. 3, which will be described in greater detail below). Typically, the gap voltage is plotted against the product of the frequency and gap separation $(f D)$. For each order of the multipactor, a pair of lines defines the region in which multipactor is possible. Given the frequency and the geometry, the curve immediately provides us with the voltage range for multipactor $\left(V_{g \min }, V_{g \max }\right)$. For zero emission velocity in a parallel plate geometry, the voltage boundaries scale as $(f D)^{2}$ and hence they appear as straight lines of slope 2 on a $\log -\log$ plot. $^{21,30}$

The voltage boundaries $V_{g \min }$ and $V_{g \max }$ can be derived by a simple integration of the force law, imposing the transit time condition $x(t=N / 2 f)=D$. This results in an equation relating the gap voltage amplitude to the external parameters $f$ and $D$, as well as the launch phase, $\theta$, and the emission energy, $E_{0}$, of secondary electrons. The orbits are stable only for a narrow range of launch phases, thus defining the voltage boundaries. The minimum voltage, $V_{g \min }$, is obtained at the maximum stable phase $\theta=\theta_{m}$ $=\arctan (2 / N \pi)$. The maximum voltage, $V_{g \max }$, is obtained at the maximum negative phase that allows an electron launched with nonzero initial velocity to reach the other plate. A negative phase is possible if the emission velocity is nonzero and the electric field changes sign before the electron hits the plate of origin. The maximum negative phase is that which results in the electron reversing, going back to the plate of origin, and reversing again just before touching that plate.

Although such curves that include the dependence of $V_{g \min }$ or $V_{g \max }$ on the emission energy, $E_{0}$, have been published, ${ }^{21}$ no explicit formulas for them have thus far been provided. For easy reference, we display, in physical units, $V_{g \text { min }}$ in Eq. $(1 \mathrm{a})^{44}$ for general emission energy $E_{0}$, and $V_{g \max }$ in Eq. (1b) only for the special case $E_{0}=0$ (therefore we call it $\left.V_{g \max 0}\right):^{30}$

$$
\begin{aligned}
& V_{g \min }=\frac{22480(f D)^{2}-(N \pi f D) \sqrt{44960 E_{0}}}{\sqrt{(N \pi)^{2}+4}}, \\
& V_{g \max 0}=\frac{22480}{N \pi}(f D)^{2},
\end{aligned}
$$

where the voltages are in volts, the frequency $f$ is in $\mathrm{GHz}$, the gap width $D$ is in cm, and the secondary-electron emission energy $E_{0}$ is in $\mathrm{eV}$. There is no simple closed-form solution of $V_{g \max }$ for nonzero $E_{0}$, but it is possible to solve for it numerically. Gill and von Engel had solved the resulting transcendental equations, expressing the maximum negative phases in terms of their " $k$ " parameter. ${ }^{22}$

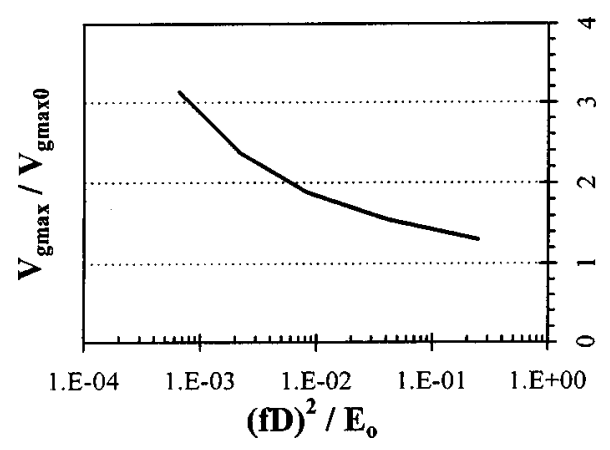

FIG. 2. Universal curve to determine the upper limiting voltage, $V_{\text {gmax }}$, for a first-order $(N=1)$, two-surface multipactor assuming a general nonzero monoenergetic emission energy $E_{0}$ (in $\mathrm{eV}$ ). Here, $f$ is the frequency in $\mathrm{GHz}$ and $D$ is the gap separation in $\mathrm{cm}$.

Reformulating the results of Gill and von Engel, we show in Fig. 2 the correction to Eq. (1b) introduced by the nonzero initial velocity. ${ }^{19}$ Note that this is valid only for a first-order $(N=1)$, two-surface multipactor. With this graph, it becomes a simple matter to calculate the upper bound $V_{g \text { max }}$ in the case of a nonzero initial velocity. First, one uses Eq. (1b) to calculate $V_{g \max 0}$ for a zero initial velocity. Next, one locates the point on the graph in Fig. 2 that corresponds to the given frequency, gap separation, and monoenergetic nonzero initial velocity, then reads off the correction factor. Multiplying this correction factor by $V_{g \max 0}$ gives the desired upper bound. Note from Fig. 2 that the importance of a nonzero initial velocity decreases for higher $(f D)$ products. This is a direct result of the higher voltages and, hence, the higher impact energies at higher $(f D)$ products. Figure 3 is an example of a susceptibility curve showing the resulting voltage boundaries for a first-order multipactor as pairs of diagonal lines, one for each of a number of monoenergetic initial velocities, $v_{0}$.

In addition to the boundaries on the voltage presented above, another factor (which has been largely ignored in the literature thus far) further constrains the existence of twosurface multipactor. This originates from the consideration that the secondary electron yield must be greater than unity

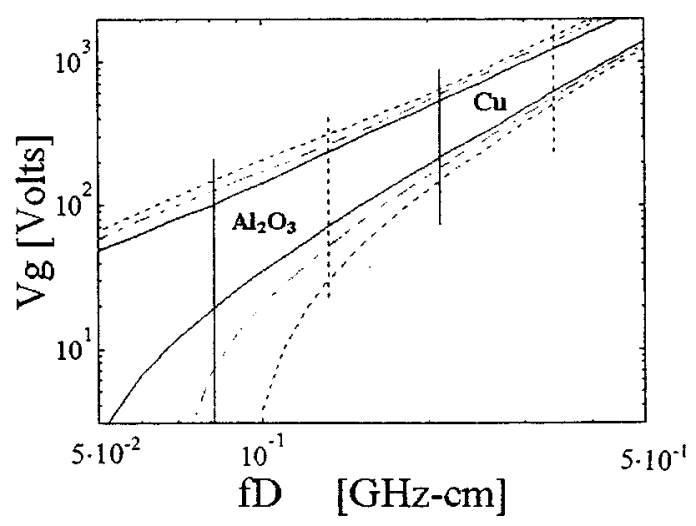

FIG. 3. Susceptibility curve for a two-surface multipactor showing the effects of nonzero emission velocity and surface materials. This example is constructed for oxygen-free copper $\left(\delta_{\max }=1.3 ; E_{\max }=600 \mathrm{eV}\right)$ and alumina $\left(\delta_{\max }=6.5 ; E_{\max }=1300 \mathrm{eV}\right)$, assuming a monoenergetic emission energy, $E_{0}=2 \mathrm{eV}(-) ; 5 \mathrm{eV}(-\cdot-)$; and $10 \mathrm{eV}(\ldots)$. 
for the avalanche to occur, and the impact energy has to be in the vicinity of $E_{1}$ (Ref. 28), even for voltages in the range $\left(V_{g \min }, V_{g \max }\right)$. Since $E_{1}$ depends on the surface materials, these surface materials provide additional conditions on the existence of multipactor. ${ }^{30}$ The first such condition is that the starting impact energy cannot be below $E_{1}$ (or else $\delta<1$ ). The second condition on the impact energy arises from dynamic considerations. Our extensive simulations (with $Q$ in the range $10-1000$ and $\delta_{\max }=1.2$ ) show that if the starting impact energy is greater than about $1.33 E_{1}$ (depending on many particulars), then cavity loading alone is insufficient in saturating the multipactor, and the discharge appears sporadically. It is possible in such a situation for the multipactor to saturate by space charge debunching, as investigated by Vaughan ${ }^{21}$ and in greater depth by Riyopoulos. ${ }^{27}$ Unfortunately, the latter papers completely neglect the effect of loading, which would in fact be significant in the intense space charge.

We now proceed to translate these boundaries onto the susceptibility curve. Examples for oxygen-free copper and alumina are provided in Fig. 3. Ignoring any nonzero initial velocity of secondaries, the impact energy can be related to the driving voltage (in the absence of multipactor) by solving the force law for the impact energy. When the gap voltage is at the upper voltage boundary $\left(V_{g 0}=V_{g \max 0}\right.$ and $\left.\theta=0\right)$ electrons experience the maximum possible impact energy. Setting that equal to $E_{1}$ gives the first boundary, the solid vertical line on the susceptibility curve (see Fig. 3). Similarly, the lower voltage boundary, at $V_{g \min }$ and $\theta_{\min }$, results in the minimum possible impact energy. Setting that to $1.33 E_{1}$ gives the second boundary, which we represent with the dotted vertical line in Fig. 3. The boundaries imposed by a given material are then ${ }^{30}$

$$
0.15 \cdot N \sqrt{\frac{E_{1}}{100}}<f D<0.17\left(N+\frac{4}{N \pi^{2}}\right) \sqrt{\frac{E_{1}}{100}},
$$

where $f$ is in $\mathrm{GHz}, D$ in $\mathrm{cm}$, and $E_{1}$ in $\mathrm{eV}$. These susceptibility curves, derived from a dynamic theory, show the qualitative features of the experimentally observed data. ${ }^{19}$

\section{B. Saturation}

Once the conditions for existence of multipactor are satisfied, any seed electron can trigger the discharge. The avalanche of electrons cannot go on unchecked, however, and sooner or later a saturation mechanism will set in. As shown in Sec. II, prior researchers have proposed space charge forces to be the (only) saturation mechanism. ${ }^{16-18,21,27}$ While this is a likely mechanism in some cases, the loading of $\mathrm{rf}$ structures by multipactor can play a significant role, especially in resonant structures. We have proposed a model of the multipactor discharge that includes such interaction with the structure. ${ }^{28}$ In this model, which is illustrated in Fig. 4, a single infinitesimally thin electron sheet travels in one dimension inside a gap between two parallel plates. Through Ramo's theorem, ${ }^{45}$ the motion of the electrons induces a wall current, $I_{m}(t)$, which in turn loads the rf structure, represented in our model by a RLC circuit with a quality factor $Q$.

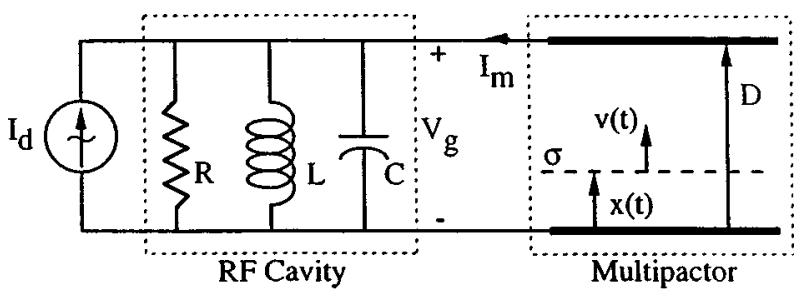

FIG. 4. Model of a two-surface multipactor and interaction with rf circuit.

The motion of the electron sheet is driven by the modified $\mathrm{rf}$ voltage across the gap, as well as by the evolving image space charge forces.

We have later extended this model to account for the mutual repulsion between the space charges through the addition of a second electron sheet. ${ }^{29}$ The two-sheet model has revealed a novel phase-focusing mechanism caused by the dependence of the secondary electron yield on impact energy (Fig. 1). This phase-focusing mechanism results in the charge density of one sheet growing at the expense of the other in our two-sheet model, thus focusing the electrons into a narrow bunch despite their mutual repulsion. This demonstrates the adequacy of the single-sheet model, at least for the regime $Q \gtrsim 10, \delta_{\max } \leq 1.5$ that we have studied.

Extensive simulations based on our one-dimensional (1-D) model show that the interaction of the multipactor with the rf circuit, a combination of detuning and de- $Q$-ing, is the dominant saturation mechanism for resonant $\mathrm{rf}$ structures $(Q \geq 10)$. The impact energy in the steady state is equal to the first cross-over point energy, $E_{1}$, of the surface material. The simulations have been augmented with an analytical derivation of the steady-state power dissipated in multipactor (for a resonantly driven, first-order discharge, the most predominant case). ${ }^{30}$ The formula shown in Eq. (3) gives the fraction of rf power consumed by the steady-state multipactor as a function of external parameters. This agrees well with the simulation results and predicts that anywhere from $1 \%-50 \%$ of the input rf power can be dissipated in the multipactor:

$$
\frac{P_{m}}{P_{d}}=\frac{-B+\sqrt{B^{2}-4 A C}}{2 A} \cdot \frac{\bar{\mu}}{\bar{r}},
$$

where

$$
\begin{aligned}
& A=\frac{1}{4}\left(\bar{\mu}-\bar{\nu}_{0}\right)^{2}+\left[\frac{1}{2}-\left(\frac{\pi}{4}-\frac{2}{\pi}\right)\left(\bar{\mu}+\bar{\nu}_{0}\right)\right]^{2}, \\
& B=\frac{2}{\pi}\left(\bar{\mu}^{2}-\bar{\nu}_{0}^{2}\right), \\
& C=\left[\frac{1}{2}-\frac{\pi}{4}\left(\bar{\mu}+\bar{\nu}_{0}\right)\right]^{2}+\frac{1}{4}\left(\bar{\mu}-\bar{\nu}_{0}\right)^{2}-\bar{r}^{2} .
\end{aligned}
$$

Here, $P_{m}$ is the power consumed by the steady-state multipactor, $P_{d}$ is the drive power, $\bar{\mu}=\left(\sqrt{2 E_{1} / m}\right) / 2 \pi f D$ is the normalized impact velocity corresponding to the first crossover point, $\bar{\nu}_{0}=\nu_{0} / 2 \pi f D$ is the normalized emission velocity of the secondaries (assumed monoenergetic), and $\bar{r}$ $=e V_{g 0} / m(2 \pi f D)^{2}$ is the normalized steady-state gap volt- 
age in the absence of multipactor. Note that the power dissipated in a multipactor discharge in the steady state is essentially a function of just two external parameters: $\bar{\mu}$, which is related to the surface material, and $\bar{r}$, which is related to the energy supplied to the structure.

Although Eq. (3) is simplified by assuming a resonantly driven cavity, our model is capable of simulating offresonance drive, and has been used to derive a more general formula for the latter case. ${ }^{46}$ Since multipactor is a resonant discharge, it can only exist for a narrow band of frequencies around the resonant frequency of the cavity. The width of this frequency band is found to be proportional to the gap voltage amplitude and inversely proportional to the $Q$ of the cavity.

The slow response time and the large amount of energy stored in a highly resonant cavity can lead to difficulties in powering such a cavity, considerably lengthening the conditioning time. Simulations with $Q \sim 1000$ show that once the voltage reaches a multipactor-prone region, it locks in as the multipactor avalanche dissipates all the additional power supplied. ${ }^{30}$ These simulations corroborate experimental observations since, in practice, it typically takes hours or days for the surface to become sufficiently conditioned to quench the multipactor and allow the voltage to rise to its full design value.

Gopinath et al. subsequently used a 1-D PIC code to simulate multipactor in a resonant cavity. ${ }^{36}$ Their more sophisticated simulations verify the predictions of our simple model, namely that cavity loading by the multipactor can result in saturation with an average impact energy in the vicinity of the first cross-over point. Moreover, they have concluded that a full distribution of initial velocities produces the same results as a monoenergetic velocity that is the average of such a distribution, ${ }^{36}$ thus validating our assumption.

\section{SINGLE-SURFACE MULTIPACTOR ON DIELECTRICS}

In contrast to metal surfaces, dielectric surfaces are capable of supporting a distribution of fixed charges. The presence of fixed charges creates dc electric fields which change the relationship between the electron motion and the rf. In this section, we focus on a situation where the rf electric field, $E_{\text {rf0 }} \sin (2 \pi f t+\theta)$, is parallel to a dielectric surface (as is usually the case near transmission windows). ${ }^{1,40}$ We assume that the surface has a net positive charge which sets up a restoring dc electric field, $E_{\mathrm{DC}}$ (Fig. 5). In addition, we use Monte Carlo methods to simulate a realistic distribution of initial velocities for the secondaries. As will be seen, the susceptibility curve and the saturation mechanism in this case are very different from those of the two-surface multipactor discussed in Sec. III.

\section{A. Susceptibility curve}

Electrons emitted from the surface with a random initial velocity and angle will be attracted back to the surface by the dc electric field (Fig. 5). During transit, they gain energy from the rf electric field and so impact the surface at an

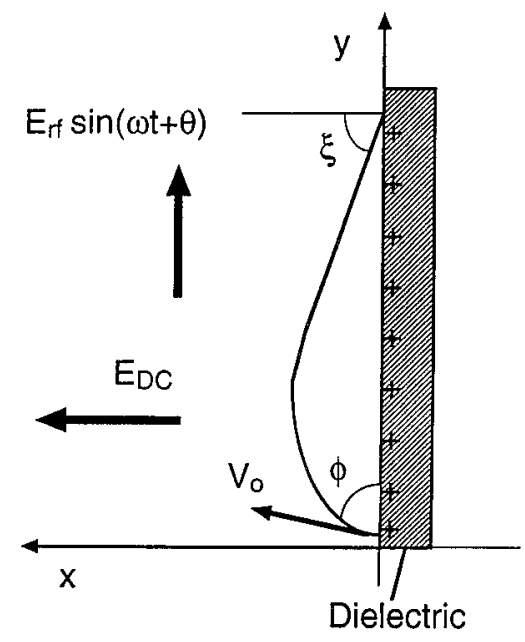

FIG. 5. Model of a single-surface multipactor in a parallel rf and normal dc electric fields.

energy higher than the emission energy. Their impact energy and angle, both of which determine the yield, depend on the phase of the rf and the random initial velocity and angle. The Monte Carlo simulation tracks a macroparticle over a large number of transits, and for every transit assigns it a random initial velocity and angle according to realistic distributions. ${ }^{40}$ Over a long time, we can see an average trend of growth or decay in the charge density of that macroparticle, depending on the external parameters of the simulation: $E_{\mathrm{rf0}}, E_{\mathrm{DC}}$, the frequency, and the material used.

With the aid of such Monte Carlo simulations, we were able to construct a universal susceptibility curve (Fig. 6) showing the regions susceptible to multipactor for any combination of external parameters and for any dielectric material. In Fig. 6, the dc electric field is plotted on one axis and the rf electric field on the other. The fields are normalized to the frequency of the $\mathrm{rf}$ and also to $E_{\max }$, a property of the surface material. The figure displays a set of boundaries, a pair for each value of $\delta_{\max 0}$ (the value of $\delta_{\max }$ for normal incidence) of the surface material, inside of which multipac-

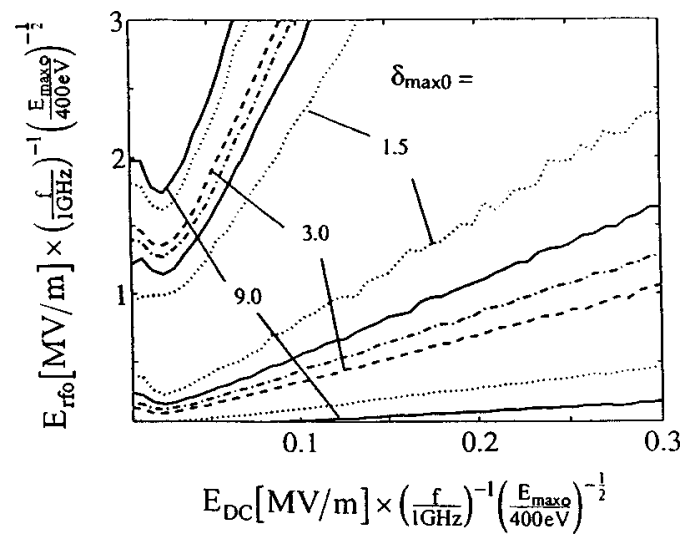

FIG. 6. Universal susceptibility curve for a single-surface multipactor on a dielectric as a function of the dc and rf electric fields, shown for various values of $\delta_{\max 0}$ (from the innermost boundaries, $\delta_{\max 0}=1.5,2.0,2.5,3.0$, 6.0 , and 9.0). This assumes a distribution of emission energies for secondary electrons, peaked at an energy $E_{o m} / E_{\max 0}=0.005$. 
tor grows. This curve can be quite useful in designing microwave components to avoid multipactor. Furthermore, as will be shown shortly, this curve can be used to predict the saturation level of multipactor and the amount of energy dissipated, and therefore the heating of the dielectric due to multipactor.

Physically, such a curve is simple to understand. The electrons gain their energy from the rf. Hence, if the rf electric field is too low (high), the average impact energy of electrons will become below $E_{1}$ (above $E_{2}$ ), and (in both cases) the yield will drop to below unity. Hence the lower boundary corresponds to the first crossover point, and the upper boundary corresponds to the second crossover point. Since the dc electric field is the restoring force, increasing it reduces the transit time and, consequently, the energy gained from the rf. Therefore the rf field must linearly increase with an increasing dc field to maintain the same yield. Finally, as $\delta_{\max 0}$ increases, the range of impact energies for which $\delta$ $>1$ widens, and so does the multipactor region.

\section{B. Saturation}

In constructing the susceptibility curve (Fig. 6) we have assumed that the charge on the window, and therefore the dc electric field, is static and unchanging. In reality, the secondary emission process of the multipactor itself creates additional surface charging. A fully dynamic model has to account for such variations in the surface charge density, as well as for the changing rf field due to the loading by the multipactor. Such a model has been very recently developed $^{41}$ and used to predict the dynamic evolution and saturation of multipactor on a dielectric. The loading of the $\mathrm{rf}$ is accounted for using a transmission line model. The surface charge responsible for the dc electric field is now broken into two parts: a small fixed charge necessary to initiate the multipactor, and a time-varying part that is created by the multipactor secondaries leaving the surface. Space charge forces between the multipactor macroparticle and the surface are included in this model.

It is evident that there are two mechanisms responsible for saturation (Fig. 7). Loading of the rf changes only the rf electric field and is indicated on the susceptibility diagram as a movement along the vertical line from A to C in Fig. 7. The space charge forces and the dynamic growth of the surface charge density correspond to a change in the dc electric field rather than the rf, and are indicated by a movement on the horizontal line from A to B. Since saturation is a combination of these two effects, it must occur at the lower boundary, where the impact energy averages around the first crossover point. Simulations have revealed that the effect of the loading is very small in this case (the dielectric window is a nonresonant structure), and therefore saturation occurs near point B. These simulations, which follow the dynamic evolution, confirm the validity of the susceptibility curves derived from the kinematic model (Fig. 6).

It is now straightforward to estimate the surface charging of the dielectric, as well as the power deposited on the dielectric surface, by the multipactor. The remarkable result is that this power is independent of most external parameters

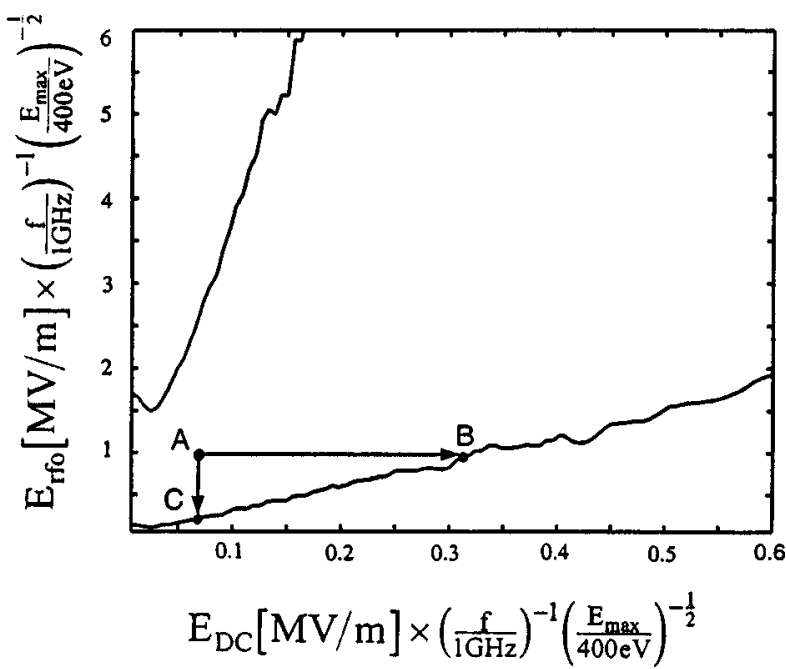

FIG. 7. Evolution of a single-surface multipactor on a dielectric. Point A designates an initial combination of electric fields that permit multipactor growth. At saturation, point B is reached.

(only weakly dependent on the details of the distribution of emission energies) and is of the order of $0.5 \%-1 \%$ of the rf power. ${ }^{41}$ Yet this amount can be sufficient to overheat the window and lead to breakage, and the results of this theory compare favorably with experimental measurements. ${ }^{35,41}$

\section{FURTHER DISCUSSION AND CONCLUSION}

\section{A. Comparison of multipactor on dielectrics to that on metals}

It is instructive now to contrast the two cases of twosurface multipactor on metals, and single-surface multipactor on a dielectric. Although in both cases the discharge is found to saturate with an impact energy in the vicinity of the first cross-over point, $E_{1}$, the saturation mechanisms are quite different. Resonant cavities are quite sensitive to loading and detuning by the multipactor, which eventually lead to its saturation. The large amount of energy and slow response time make the passage of the cavity voltage through narrow multipactor bands very difficult, as multipactor can readily grow to a large extent and "lock in" the voltage. Dielectric windows, on the other hand, are much less sensitive to such loading and saturate primarily by space charge forces. The proximity of the charges to the dielectric surface, unlike the two-surface configuration, further aids the space charge forces. The most striking difference, however, is the absence of the resonance condition on the transit time in the singlesurface case. This greatly widens the parameter space for multipactor and makes the dielectric surface much more susceptible. Even though the power dissipated by multipactor on a dielectric window is relatively small, the poor heat conductivity of dielectrics possibly makes it sufficient to destroy the window.

\section{B. Summary}

In light of the historical development of multipactor theory, the theories presented in this paper survey the first attempt on multipactor susceptibility curves and saturation 
from dynamic considerations, taking into account the interaction with the surrounding $\mathrm{rf}$ structure and the material properties of the surface. Computer simulations have revealed the time evolution of the discharge, the dependence on materials, a new phase-focusing mechanism among multiple electron sheets, and the relative importance of the various saturation mechanisms. The results of this study are well in agreement with experimental observations.

\section{ACKNOWLEDGMENTS}

In the development of these theories, we have benefited from conversations with C. M. Armstrong, J. Benford, C. K. Birdsall, M. Brennan, K. Brown, D. Chernin, W. Getty, V. P. Gopinath, E. Haebel, J. Haimson, J. P. Holloway, S. Isagawa, T. Lavine, A. Lombardi, F. Mako, R. Miller, R. Poirier, R. Rimmer, S. Riyopoulos, J. Shiloh, J. J. Song, G. Thomas, J. P. Verboncoeur, J. Wang, and P. Wilson.

This work was supported by the Multidisciplinary University Research Initiative (MURI), managed by the Air Force Office of Scientific Research and subcontracted through Texas Tech University, by the Department of Navy Grant N00014-97-1-G001 issued by the Naval Research Laboratory, by the Northrop Grumman Industrial Affiliates Program, and also by the Department of Energy.

${ }^{1}$ D. H. Preist and R. C. Talcott, IRE Trans. Electron Devices ED-8, 243 (1961).

${ }^{2}$ J. R. M. Vaughan, IEEE Trans. Electron Devices ED-8, 302 (1961).

${ }^{3}$ A. S. Gilmore, Microwave Tubes (Artech House, Norwood, MA, 1986), p. 474.

${ }^{4}$ S. Yamaguchi, Y. Saito, S. Anami, and S. Michizono, IEEE Trans. Nucl. Sci. 39, 278 (1992).

${ }^{5}$ Y. Saito, S. Michizono, S. Anami, and S. Kobayashi, IEEE Trans. Electr. Insul. 28, 566 (1993).

${ }^{6}$ J. Tuckmantel, C. Benvenuti, D. Bloess, D. Boussard, G. Geschonke, E. Haebel, N. Hilleret, S. Juras, H. P. Kindermann, J. Uythoven, C. Wyss, and M. Stirbet, IEEE Proceedings of the 1995 Particle Accelerator Conference (Institute of Electrical and Electronics Engineers, Piscataway, NJ, 1995), p. 1642.

${ }^{7}$ Linear Accelerators, edited by P. M. Lapostolle and A. L. Septier (North Holland, Amsterdam, 1970), p. 917.

${ }^{8}$ K. J. Kleman, Proceedings 1993 Particle Accelerator Conference (Institute of Electrical and Electronics Engineers, Piscataway, NJ, 1993), p. 924.

${ }^{9}$ J. R. M. Vaughan, IEEE Trans. Electron Devices ED-15, 883 (1968).

${ }^{10}$ M. Yoshida, S. Isagawa, Yo. Takeuchi, M. Ono, M. Sato, K. Takata, and H. Baba, Proceedings 6th Symposium on Accelerator Science and Technology, Tokyo, Oct. 1987 (Iomico, Tokyo, 1987), pp. 126-128.

${ }^{11}$ E. G. Schweppe, R. Bachmor, and E. Demmel, IEEE Proc. of the 1993 Particle Accelerator Conference (Institute of Electrical and Electronic Engineers, Piscataway, NJ, 1993), p. 1178.

${ }^{12}$ F. Hohn, W. Jacob, R. Beckmann, and R. Wilhelm, Phys. Plasmas 4, 940 (1997).

${ }^{13}$ P. F. Clancy, Microw. J. 77 (March, 1978).

${ }^{14}$ A. D. Woode and J. Petit, Microw. J. 142 (January, 1992).

${ }^{15}$ N. Rozario, H. F. Lenzing, K. F. Reardon, M. S. Zarro, and C. G. Baran,
IEEE Trans. Microwave Theory Tech. MTT-42, 558 (1994).

${ }^{16}$ W. J. Gallagher, Proc. IEEE 94 (Jan. 1969).

${ }^{17}$ W. J. Gallagher, IEEE Trans. Nucl. Sci. NS-26, 4280 (1979).

${ }^{18} \mathrm{~F}$. Mako and W. Peter, IEEE Proceedings of the 1993 Particle Accelerator Conference 2702 (Institute of Electrical and Electronics Engineers, Piscataway, NJ, 1993).

${ }^{19}$ R. A. Kishek, Ph.D. dissertation, University of Michigan, Ann Arbor, MI, 1997.

${ }^{20}$ A. J. Hatch and H. B. Williams, J. Appl. Phys. 25, 417 (1954); theory later modified in A. J. Hatch and H. B. Williams, Phys. Rev. 112, 681 (1958).

${ }^{21}$ J. R. M. Vaughan, IEEE Trans. ED-35, 1172 (1988).

${ }^{22}$ E. W. B. Gill and A. von Engel, Proc. R. Soc. London, Ser. A 192, 446 (1948).

${ }^{23}$ S. Brown, Basic Data of Plasma Physics (American Institute of Physics, New York, 1959, reprinted 1994), p. 202-221.

${ }^{24}$ A. L. Gilardini, J. Appl. Phys. 78, 783 (1995).

${ }^{25}$ S. Riyopoulos, D. Chernin, and D. Dialetis, Phys. Plasmas 2, 3194 (1995).

${ }^{26}$ S. Riyopoulos, D. Chernin, and D. Dialetis, IEEE Trans. Electron Devices ED-44, 489 (1997).

${ }^{27}$ S. Riyopoulos, Phys. Plasmas 4, 1448 (1997).

${ }^{28}$ R. Kishek and Y. Y. Lau, Phys. Rev. Lett. 75, 1218 (1995).

${ }^{29}$ R. A. Kishek and Y. Y. Lau, Phys. Plasmas 3, 1481 (1996).

${ }^{30}$ R. A. Kishek, Y. Y. Lau, and D. Chernin, Phys. Plasmas 4, 863 (1997)

${ }^{31}$ J. W. Noe, Nucl. Instrum. Methods Phys. Res. A 328, 291 (1993).

${ }^{32}$ D. Proch, D. Einfeld, R. Onken, and N. Steinhauser, IEEE Proceedings of the 1995 Particle Accelerator Conference (Institute of Electrical and Electronics Engineers, Piscataway, NJ, 1995), p. 1776.

${ }^{33}$ C. M. Lyneis, H. A. Schwettman, and J. P. Turneaure, Appl. Phys. Lett. 31, 541 (1977).

${ }^{34}$ C. Pasotti, P. Pittana, and M. Svandrlik, Proceedings of the IEEE 1997 Particle Accelerator Conference Vancouver, to be published (Institute of Electrical and Electronics Engineers, Piscataway, NJ).

${ }^{35}$ Robert Alan Rimmer, Ph.D. dissertation, Lancaster University, Lancaster, UK, 1988.

${ }^{36}$ V. P. Gopinath, J. P. Verboncoeur, and C. K. Birdsall, "Multipactor electron discharge physics using an improved secondary emission model," submitted to Phys. Plasmas.

${ }^{37}$ R. Woo and A. Ishimaru, J. Appl. Phys. 38, 5240 (1967).

${ }^{38}$ R. Woo, J. Appl. Phys. 39, 1528 (1968).

${ }^{39}$ E. Somersalo, P. Yla-Oijala, and D. Proch, IEEE Proceedings of the 1995 Particle Accelerator Conference (Institute of Electrical and Electronics Engineers, Piscataway, NJ, 1995), p. 1500.

${ }^{40}$ R. A. Kishek and Y. Y. Lau, Phys. Rev. Lett. 80, 193 (1998).

${ }^{41}$ L. K. Ang, Y. Y. Lau, R. A. Kishek, and R. M. Gilgenbach, "Power deposited on a dielectric by multipactor," to appear in IEEE Trans. Plasma Sci. (1998).

${ }^{42}$ C. K. Birdsall and W. B. Bridges, Electron Dynamics of Diode Regions (Academic, New York, 1966); also O. Hachenberg and W. Brauer, Adv. Electron. Electron Phys. XI, 413 (1959).

${ }^{43}$ J. R. M. Vaughan, IEEE Trans. Electron Devices ED-36, 1963 (1989); verified experimentally in A. Shih and C. Hor, IEEE Trans. Electron Devices ED-40, 824 (1993).

${ }^{44}$ We remark that Eq. (1a) is not exact for nonzero initial velocity, because in such a case the maximum stable phase $\theta_{m}$ is no longer given by the simple expression $\arctan (2 / N \pi)$ derived for zero emission velocity, but is nevertheless sufficiently accurate.

${ }^{45}$ S. Ramo, Proc. IRE 27, 584 (1939); also W. Shockley, J. Appl. Phys. 9, 635 (1938).

${ }^{46}$ August Valfells, R. A. Kishek, and Y. Y. Lau, Phys. Plasmas 5, 300 (1998). 